\title{
Advances in Electronic Interconnection Materials
}

\author{
C.M. GOURLAY ${ }^{1,3}$ and B. ARFAEI ${ }^{2}$ \\ 1.-Department of Materials, Imperial College London, London SW7 2AZ, UK. 2.-Ford Motor \\ Company, Dearborn, MI 48124, USA. 3.—e-mail: c.gourlay@imperial.ac.uk
}

While the transition to $\mathrm{Pb}$-free solder materials is almost complete for most electronic segments, the ongoing reliability requirements of next-generation electronics have introduced significant new challenges and triggered intensive global research. This is being driven by trends including: (1) the ongoing miniaturization and 3D integration of consumer electronics for improved performance (e.g., processor speeds and bandwidth) which leads to higher current densities and steeper temperature gradients across fine pitch joints; (2) the operation of joints in harsher environmental conditions, which can, for example, enable electronic component units (ECUs) to be moved closer to the engine, reducing wiring and, therefore, mass; (3) the move towards higher reliability electronics caused by the increased reliance on sensors in applications from security monitoring to electrical and autonomous driving; and (4) the need for reliable, low-temperature solder alloys that have economic and environmental benefits and can reduce the dynamic warpage that is necessary for the assembly of thin, flexible electronics.

Many of the challenges for the improved reliability of electronic interconnections are materials challenges. For example, new interconnection materials are required for safe operation at higher temperature, cycling through larger temperature ranges whilst enduring higher current densities, steeper temperature gradients and impact loading. These may include new solder alloys, sintering pastes, and conductive adhesives, which in turn may require new, optimized substrates, surface finishes and fluxes. At the same time, new manufacturing processes, such as new deposition methods, transient liquid phase bonding approaches, and direct $\mathrm{Cu}-\mathrm{Cu}$ bonding, are being explored and implemented. In parallel, to improve the prediction of in-service reliability and joint lifetime,

Christopher M. Gourlay is the JOM advisor for the Electronic Packaging and Interconnection Materials Committee of the Functional Materials Division. Gourlay and Babak Arfaei are the guest editors for the topic Advances in Electronic Interconnection Materials in this issue. continuum-based reliability models need to be supported by detailed crystallographic and microstructure-based models of thermo-mechanical fatigue, vibration and impact loading.

The topic Advances in Electronic Interconnection Materials aims to provide JOM readers with an update on research on electronic solder joints, and on future research requirements, as new interconnection materials enter service and operate under increasingly harsh conditions. We have three papers related to the development of improved solder alloys, the understanding of phenomena and microstructures in solder-substrate reaction zones, and the modeling of in-service reliability of solder joints.

The first paper, by Delhaise et al., focuses on bismuth additions to Sn-based solders. This paper is motivated by the resurgence of interest in $\mathrm{Bi}$ additions to $\mathrm{Pb}$-free solders due to the effectiveness of $\mathrm{Bi}$ as a solid solution strengthener and precipitation hardener, and its ability to significantly improve solder joint lifetime in thermal cycling. The authors present a comprehensive experimental study on the diffusion of $\mathrm{Bi}$ in solid $\beta$-Sn, considering the effects of crystallographic anisotropy, temperature, and high diffusivity pathways. The directional diffusivities are measured and discussed relative to the tetragonal crystal structure of $\beta$-Sn. For the $\mathrm{Bi}$ solute, the diffusivity is shown to be higher along the $c$-direction of $\beta$-Sn and the maximum and minimum diffusivities are within an order of magnitude, which is markedly different to past work on smaller solute atoms. The new understanding of $\mathrm{Bi}$ mass transport in $\beta$-Sn provides a foundation for a better understanding of the homogenizing and solutionizing of $\mathrm{Bi}$ within $\beta$-Sn at high temperature, and the precipitation and coarsening of $(\mathrm{Bi})$ plates at low temperature.

The second paper, by P.T. Vianco, overviews selected interfacial phenomena between solders and substrates, the microstructures that form during solder reactions and evolve in service, and interfacial failures. This paper begins with a 
summary of wetting in solder reactions and the role of surface coatings and surface finishes. It then uses a collection of short case studies, from the author's extensive experience of electronics packaging and reliability, to illustrate a variety of field failures. Each case study details failure analyses for solder joints/alloys and the potential failure mechanisms. The paper includes examples for $\mathrm{Sn}-37 \mathrm{~Pb}$ solder and $\mathrm{Pb}$-free solders, such as $\mathrm{Sn}-3.9 \mathrm{Au}-0.6 \mathrm{Cu}$, highlighting some important microstructural differences. An overriding theme throughout the paper is the importance of microstructure evolution in solder reaction zones on the long-term reliability of electronic interconnections.

The third article, by Clech et al., is concerned with the challenges of predicting solder joint lifetime and the use of acceleration factors. The paper overviews the key phenomena affecting reliability during thermal cycling for $\mathrm{SnPb}$ and $\mathrm{Sn}-\mathrm{Ag}-\mathrm{Cu}$ (SAC) solder alloys, and then reviews the current thermo-mechanical reliability models used to predict the lifetime of solder joints in field service. There is a focus on the successes and limitations of the models. The authors then build from their review and experience to suggest guidelines for the development of future reliability models, and discuss the need to develop microstructure-based models that account for the orientations of the small number of $\beta$-Sn grains in each joint, the presence of beach ball and interlaced $\beta$-Sn grain morphologies, and the length-scale of the eutectic intermetallics (typically $\mathrm{Ag}_{3} \mathrm{Sn}$ and $\mathrm{Cu}_{6} \mathrm{Sn}_{5}$ ), as well as their evolution in service. This suggestion can be considered a research call to arms as new high-reliability alloys and safety critical interconnects become more widespread.

We invite you to join us at the TMS meeting in San Antonio, Texas, in March 2019, where the symposium "Advanced Microelectronic Packaging, Emerging Interconnection Technology, and $\mathrm{Pb}-\mathrm{Free}$
Solder" will include such topics as lead-free solder, alternative interconnects, conductive adhesives, substrate materials, 3D packaging, wafer-level packaging, quality, reliability, and failure analysis. We also invite you to attend the 15th Annual LeadFree Solders and Interconnect Technology Workshop and the meeting of the Electronic Packaging and Interconnection Materials Committee, both at the same TMS meeting.

The following list summarizes the papers being published under the topic of "Advances in Electronic Interconnection Materials". To download any of the papers, follow the URL http://link.springer.com/jou rnal/11837/71/1/page/ 1 to the table of contents page for the January 2019 issue (vol. 71, no. 1).

- "Solid-State Diffusion of Bi in Sn: Effects of Anisotropy, Temperature, and High Diffusivity Pathways" by André Delhaise, Zhangqi Chen, and Doug D. Perovic

- "A Review of Interface Microstructures in Electronic Packaging Applications" by Paul Thomas Vianco.

- "Pb-Free Solder Joint Thermo-Mechanical Modeling: State of the Art and Challenges" by Jean-Paul M. Clech, Babak Arfaei, and Richard J. Coyle

\section{OPEN ACCESS}

This article is distributed under the terms of the Creative Commons Attribution 4.0 International License (http://creativecommons.org/licenses/by/4.0/ ), which permits unrestricted use, distribution, and reproduction in any medium, provided you give appropriate credit to the original author(s) and the source, provide a link to the Creative Commons license, and indicate if changes were made. 